\title{
PRESCRIPTION WRITING PRACTICES AMONG DOCTORS IN A TEACHING HOSPITAL IN ANDHRA PRADESH: AN OBSERVATIONAL STUDY
}

\author{
R. V.S. N. S. Ramachandrudu ${ }^{1}$
}

${ }^{1}$ Associate Professor, Department of Pharmacology, Government Medical College, Ananthapuramu, and Affiliated to Dr. NTR University of Health Sciences, Andhra Pradesh.

\section{ABSTRACT}

Prescription is a legal document that contains detailed information regarding customized pharmacological treatment designed by a clinician for a patient. Also, the patient takes his medicines seeing his prescription as his guide whenever he is in doubt. It also serves as a reference for medico legal issues. Such a legal document should be carefully written. This is an observational study done to identify and describe various aspects in prescription writing among doctors in a teaching hospital in Andhra Pradesh.

AIM

To disclose prescription writing practices among doctors in a teaching hospital in Andhra Pradesh.

\section{MATERIALS AND METHODS}

Snapshots of 100 prescriptions coming to Pharmacy of the teaching hospital were taken using a digital camera for a period of 30 days and were scrutinized to identify various elements in prescription writing.

\section{RESULTS}

All prescriptions were seen to miss some information or contain unclear information.

\section{CONCLUSION}

The standard protocol of writing prescriptions is not being followed by doctors. The major reason for this could be increased patient-doctor ratio.

\section{KEYWORDS}

Prescription Writing, Patient-Doctor Ratio, Legal Document.

HOW TO CITE THIS ARTICLE: Ramachandrudu RVSNS. Prescription writing practices among doctors in a teaching hospital in Andhra Pradesh: an observational study. J. Evolution Med. Dent. Sci. 2016;5(43):2649-2653, DOI: 10.14260/jemds/2016/620

\section{INTRODUCTION}

Prescription is a legal document that contains detailed information regarding customized pharmacological treatment designed by a clinician for a patient. It can be a reference during medico-legal issues. It also serves as a communication between clinician and pharmacist. Such an important document should be written very carefully. But, it is very common to see some missing details or unclear information in prescriptions written by doctors. Prescription writing is taught to medical students in their first clinical year along with other topics of pharmacology. Special emphasis is given to this topic by including it in practical examination (Prescription writing, criticism and correction of prescription). In spite of such careful training, acceptable standards in prescription writing are many times not seen. One of the major reasons could be the patient-doctor ratio in the hospitals. At times, a single doctor has to treat hundreds of patients in hospital. This is particularly evident in government hospitals. So the reason for such type of prescription writing may be attributed to overload of patients and so doctors should not always be condemned.

Financial or Other, Competing Interest: None.

Submission 15-04-2016, Peer Review 10-05-2016,

Acceptance 16-05-2016, Published 27-05-2016.

Corresponding Author:

Dr. R. V.S. N. S. Ramachandrudu,

G6, Block II,

Govt. Medical Staff Quarters, Jesus Nagar

Ananthapuramu-515001.

Andhra Pradesh, India.

E-mail: dr.ramchandrudu@gmail.com

DOI: $10.14260 /$ jemds/2016/620
Certain rules should be strictly followed while writing prescription for benefit of patient and also to avoid medicolegal problems. Unfortunately, a number of missing/unclear information is seen in prescriptions of many doctors. Missing or unclear information in prescriptions include absence of date, absence of signature, illegible hand writing, usage of ambiguous symbols and abbreviations which confuse the patient and so on. Among these, illegible hand writing is very commonly known problem. This can lead to disastrous situations.

This is an observational study done to identify and describe prescription writing practices among doctors in a teaching hospital in Andhra Pradesh.

\section{MATERIALS AND METHODS}

After getting approval from Institutional Ethical Committee and consent from patients, snapshots of 100 prescriptions coming to Pharmacy of teaching hospital were taken using a digital camera for a period of 30 days ( $1{ }^{\text {st }}$ March 2016 to $30^{\text {th }}$ March 2016). All these snapshots were kept very much confidential. All these prescriptions belong to patients between age groups 25 yrs. - 65 yrs. These snapshots were carefully scrutinized to identify various elements in prescription writing.

The Missing/Unclear Information in these Prescriptions Were Categorized as

1. Missing Information about Doctor/Hospital/Clinic.

a. Include doctor/hospital information, which is generally pre-printed.

b. Signature of doctor. 
2. Missing Information about patient.
a. Name.
b. Age.
c. Sex.
d. Address.
e. Presenting complaint.

3. Missing/Unclear Information regarding prescribed medicines.
a. Date.
b. Generic name.
c. Dose.
d. Duration.
e. Frequency.
f. Route of Administration.

\section{Statistics}

Descriptive statistics was used to analyse data. Results are indicated in percentages. Bar charts are used to illustrate results.

\section{RESULTS}

All prescriptions were seen to have some missing/unclear information. Missing information about patient and medication was commonly seen in this study. Sex, Address and Date were seen to be missing in most of the prescriptions. Though patient's name was present in all prescriptions, Name with Initial was mentioned in only a few prescriptions.

\section{Details are Tabulated below}

\begin{tabular}{|c|l|c|}
\hline \multicolumn{1}{|c|}{ Type of Error } & $\begin{array}{c}\text { Percentage of } \\
\text { Doctors }\end{array}$ \\
\hline I & Missing Information about & \\
& $\begin{array}{l}\text { Doctor/Hospital } \\
\text { a. Pre-printed Information }\end{array}$ \\
& missing & $4 \%$ \\
& b. Signature missing & $4 \%$ \\
\hline II & Missing Information about & \\
& patient & $0 \%$ \\
& a. Name & $0 \%$ \\
& b. Age & $94 \%$ \\
& c. Sex & $96 \%$ \\
& d. Address & $54 \%$ \\
& e. Presenting complaint & $28 \%$ \\
& f. Diagnosis & $98 \%$ \\
\hline III & g. Occupation & \\
& Missing Information about & \\
& prescribed medicines & $90 \%$ \\
& a. Date & $02 \%$ \\
& b. Frequency & $01 \%$ \\
& c. Generic name & $0 \%$ \\
& d. Route & $30 \%$ \\
\hline & e. Dose & $12 \%$ \\
\hline & f. Duration & $11 \%$ \\
\hline & g. Legibility & \\
\hline
\end{tabular}

\section{DISCUSSION}

\section{Information about Doctor or Hospital/Clinic}

All this information is pre-printed except signature of doctor. Pre-printed information includes Name of Hospital/Doctor, Qualifications, Registration Number, Contact Number, etc. MCI has made registration of private clinics/hospitals mandatory.
So details of registration of doctor/hospital should be mentioned here. These things will indicate whether he/she is allopathic practitioner or not. Pharmacists should be trained to reject prescriptions of modern system of medicine given by non-allopathic practitioners. Phone Number of doctor/hospital for communication should also be provided. This helps the patient/pharmacist to contact the doctor during any doubt. This information will be missing only when prescriptions are not written on prescription pads.

In this study, most of the prescriptions are written on prescription pads provided by hospital. Only $4 \%$ of prescriptions were not written on prescription pads. So information about doctor and hospital were missing in these prescriptions.

In a similar study.[1] by Ather A et al in Gulbargha, it was seen that significantly higher number of prescriptions (90\%) did not have prescriber's name.

\section{Signature}

The prescription is valid only if signature of doctor is present on it. Facsimile signature should never be used on prescriptions. In this study, only $4 \%$ of prescriptions lacked signature.

In the study.[2] by Vaishali D Phalke et al at Western Maharashtra, $12 \%$ prescriptions lacked signature.

\section{INFORMATION ABOUT PATIENT}

This Information is Manually Written. This Information can be Categorized as

I) Essential, and

II) Additional.

\section{Essential Information Includes}

1. Patient's Name.

2. Patient's Age.

3. Sex of the patient.

4. Complaint.

5. Diagnosis.

\section{Additional Information Includes}

1. Patient's occupation.

2. Patient's address.

In Government and Corporate Hospitals, Patient's information like Name, Age, Sex, Weight, Address, Occupation is generally entered by Doctor's assistant (Receptionist). However, it is doctor's responsibility to check the entered information. Diagnosis and medical history will be entered by doctor.

The percentage of flaws in "Information about Patient" in this study is depicted in the graph.

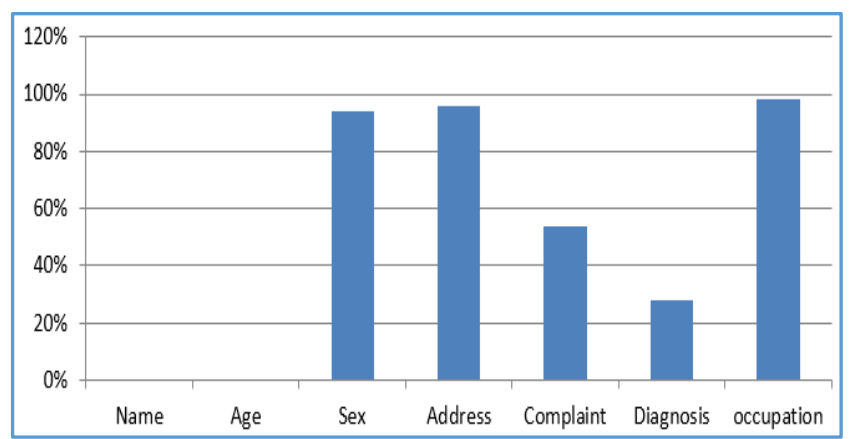

Fig. 1: Graph Depicting \% of Prescriptions Missing Various Variables in Patient Information 
Patient's Name, this should be written along with surname because it avoids misdistribution of drugs in pharmacy when 2 patients with same name come. In this study though name was mentioned in all prescriptions, only $8 \%$ of prescriptions had name along with surname.

In a similar study.[3] it was seen that name of the patient is mentioned in all prescriptions. On contrary, in a study.[4] involving final year medical students in Nigeria it was seen that patient's name was present only in $43.39 \%$ of prescriptions.

\section{Patient's Age}

Ideally, dose has to be determined based on patient's age. In this study, all prescriptions contained Age of the Patient. Similarly in another study.[5] by Sneha Kumari et al at Ranchi, all the prescribers mentioned patient's name and age.

\section{Sex of the Patient}

In this study most of the prescriptions (94\%) did not contain sex of the patient. In a similar study.[6] sex of the patient was mentioned only in $33.3 \%$ of prescriptions. It is presumed that sex is understood by the patient's Name. However, there are number of examples where a Name is suitable to both genders. So mentioning sex of the patient gives uniqueness to the prescription.

\section{Patient's Occupation}

Occupation indicates certain diseases, e.g.: -Asbestosis in people working in mines, occupational asthma in workers of cigarette factory. It also gives an idea whether the patient is affordable to buy costly brand medicines if necessary. Also, dexterity and compliance for taking medicines can be estimated by patient's occupation. In this study, occupation was not mentioned in $98 \%$ of prescription.

\section{Patient's Address}

In this study, Address was not mentioned in $96 \%$ of prescriptions which is very important for identifying endemic diseases like Malaria (In hill areas) and fluorosis (Common in certain districts like Nalgonda). It is also important for referring the patient to nearby Physician/Health Centre for follow-up.

\section{Presenting Complaint}

This has to be noted in patient's own words. Medical words should not be substituted for explaining patient's complaint. In this study, $48 \%$ of prescriptions lacked complaint. Eg:- Should not record pain of joints as "Arthritis."

\section{Diagnosis}

In this study, only $28 \%$ of prescriptions were without diagnosis. In $14 \%$ of prescriptions, it was seen that diagnosis was mentioned under the heading "Complaint." It was also seen that "?" was not indicated for provisional diagnosis and investigations were simply advised. In Tamil Nadu study.[7] it was seen that good number of doctors mentioned diagnosis on the prescriptions.

\footnotetext{
Information about Medication, this Includes

1. Date of prescription.

2. Dose of drug.

3. Frequency at which drugs are to be taken.

4. Name of the Drug.

5. Route of Administration.
}

The percentage of flaws in "Information about Medication" in this study is depicted in the graph.

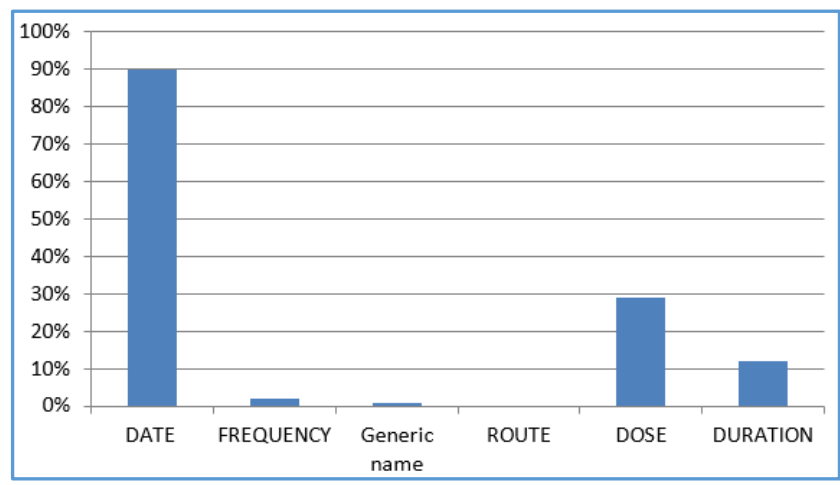

Fig. 2: \% of Missed Components in "Information about Medication"

All this information should be entered by doctor (Excepting date, which can be entered by assistant). Doctor's assistant should never be allowed to enter information about medication on prescription pad.

\section{Date of Prescription}

Before writing the medications, it is very important to mention the date of writing the prescription. It gives information about how long patient is using the medication. Pharmacist gets an idea about validity of prescription seeing the date. This is particularly important in case of abuse drugs like drugs acting on CNS, where the prescription has to be reviewed at intervals. In this study, it was seen that date was not mentioned in $90 \%$ prescriptions. (However, serial number was mentioned in all prescriptions from which date can be identified when needed). On contrary, a study.[8] in Mahaboobnagar revealed that date was present in most prescriptions (94.4\%).

\section{Name of the Drug}

Ideally, the generic name of the drug has to be written in an acceptable handwriting. Abbreviations of drugs should not be used. In this study, abbreviations are used in $57 \%$ of prescriptions. In a similar study.[9] it was seen that abbreviations were used in significant number (69.8\%) of prescriptions.

MCI has advised doctors to write the names of drugs in capitals in a legible handwriting. In this study, none of the Prescriptions were written in Capitals and $11 \%$ of prescriptions were illegible. In a similar study.[10] it was seen that most of the prescriptions were written in lower case letters.

To the extent possible, it is always better to write generic names of drugs. But generic names of drugs are rarely used by private practitioners. However, in this study, only $14 \%$ of prescriptions contained brand names and most of the Doctors were using Generic names of Drugs. On contrary, a study.[11] in Guntur revealed that very few prescriptions contained generic names of drugs. In Maharashtra study.[12] it was seen that only $60 \%$ of drugs were prescribed by generic name. In another study.[13] it was seen that most of the prescriptions lacked generic names of drugs.

Using brand names of drugs may be ambiguous. In a study.[14] by PV Rataboli et al, it was concluded that several brand names were strikingly similar. There was a report.[15] 
when pharmacist got confused and dispensed wrong medicine which resulted in allergic dermatitis to the patient.

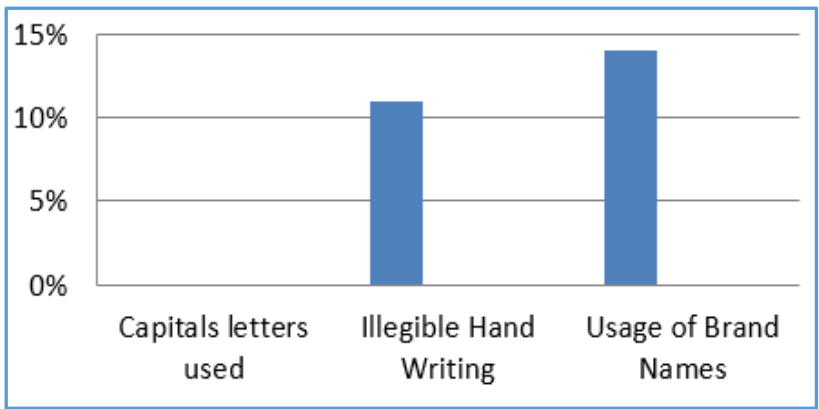

Fig. 3: Graph Depiction Errors in Writing Name of Drug in Prescription

\section{Route of Administration}

Certain drugs are available in many different routes like oral, sublingual, parenteral, dermal, etc. So it is important to clarify the pharmacist what form of drug has to be dispensed. For eg:If angina patient is prescribed sublingual Nitroglycerin (Without mentioning route of administration), there is a chance for pharmacist to dispense Oral Nitroglycerin. Then, whenever an attack occurs, onset of action of oral nitroglycerin will be comparatively late. This may be dangerous to patient. Hence, clinicians must make sure to mention the route of drug for the benefit of patients. Similarly, Bisacodyl suppositories when prescribed, route of administration should be mentioned.

In this study, it was seen that Route of Administration was mentioned in all Prescriptions. On contrary, a study.[16] done in Tirupathi revealed that route was mentioned only in $32.1 \%$ of prescriptions.

\section{Dose}

Ideally dosage has to be calculated according to Weight, Age and systemic condition (Liver and Kidney functioning) of the patient. Dose should be clearly mentioned with units. There are many drugs, which are available in different strengths. Use of over dose or under dose will result in undesirable outcomes. In this study, $30 \%$ of prescriptions lacked dose of drug. In another study.[17] it was seen that only $8.7 \%$ of prescriptions lacked dose of drug.

\section{Frequency}

In this study, only $2 \%$ of Prescriptions lacked Frequency. Frequency at which drug has to be taken is mentioned symbolically. These symbols should not confuse the patients. But there is no generalized usage of these symbols by all doctors. Such usage of symbols by clinicians was seen in this study too. For eg:- For prescribing diclofenac - $50 \mathrm{mg} 2$ times a day for 3 days, different doctors use symbols which indicate a different meaning.

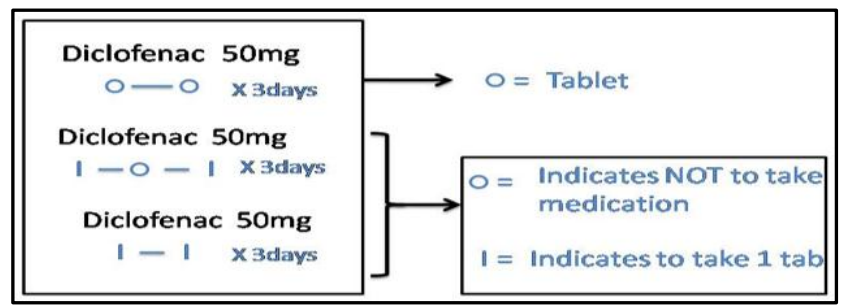

Hence, there should be a universal system of symbols (TID/TDS; BID/BD; QID, OD; H.S etc.) in prescription writing which was taught in Pharmacology and can be easily understood by all doctors, pharmacists and can be easily explained to the patients. In a study.[18] it was seen that $30 \%$ of dental prescriptions contained error prone abbreviations, symbols and dose designations. A study.[19] in Rajasthan revealed significant number of prescriptions (21\%) without clear instruction to pharmacist.

\section{Duration}

In this study, only $11 \%$ of prescriptions lacked duration. For other drugs like benzodiazepines, psychotropics, antidepressants, etc. It is imperative to mention Duration of drug usage. A study.[20] in East Godavari revealed that duration was not mentioned in $46 \%$ of prescriptions, which is higher than this study.

\section{CONCLUSION}

The standard protocol for writing prescriptions is not being followed by doctors. One of the major reasons could be the doctor - patient ratio in the hospitals. At times, a single doctor has to treat hundreds of patients in government hospitals. Also prescription writing should be taught to undergraduates in ethical point of view to minimize errors and the physicians must be made aware of its importance. In our primary school teaching, our teachers advised to write copy writing to develop good hand writing, but as we promote to higher classes we neglect the hand writing leading to illegible handwriting, same is with prescription writing. We do not follow what we have been taught in Pharmacology.

\section{REFERENCES}

1. Ather A, Neelkantreddy P, Anand G, et al. A study on determination of prescription writing errors in outpatient department of medicine in a teaching hospital. Indian Journal of Pharmacy Practice 2013;6(2):21-4.

2. Phalke VD, Phalke DB, Syed MM, et al. Prescription writing practices in a rural tertiary care hospital in western Maharashtra, India. The australian medical journal 2011;4(1):4-8.

3. Sunitha J, Wasim Raja S, Sravan Kumar Y, et al. Assessment of prescriptions for its completeness in India-a prospective study. International journal of Pharmacotherapy 2013;3(1):34-8.

4. Oshikoya KA, Bello JA, Ayorinde EO. Prescribing knowledge and skills of final year medical students in Nigeria. Indian journal of Pharmacology 2008;40(6):2515.

5. Sneha Kumari, Haider S, Kashyap V, et al. A study on pattern of prescription writing practices at rajendra institute of medical sciences, Ranchi. Indian journal of preventive and social medicine 2014;45(1-2).

6. Amira E Abdellah, Sirageldin MK Abdelrahman. Prescription writing quality in paediatric teaching hospitals in Khartoum. Sudanese Journal Of Paediatrics 2012;12(1):64-9.

7. Sekharan Gopalakrishnan, Parasuraman Ganeshkumar, Ajitha Katta. Assessment of prescribing practices among urban and rural general practitioners in Tamil Nadu. Indian Journal of Pharmacology 2013;45(3):252-7. 
8. Raj Kishore Mahato, Parineeta Suman. Analysis of medical prescription: prescriber's adherence to the basic principles of prescription order writing. International journal of science and technology 2013;15(1):1042-9.

9. Ranjeeta Kumari, Idris MZ, Vidya Bhushan, et al. Assessment of prescription pattern at public health facilities of lucknow district. Indian journal of Pharmacology 2008;40(6):243-7.

10. Sudheer G, Srinivasan K, Ravi Prabhu G. A study of prescription writing practices of doctors in medical units in a teaching hospital. J of Drntr univ of health sciences 2013;2(1):22-4.

11. Simpson GB, Naveen Chaudhary G. Comparative analysis of prescription writing by teaching and non-teaching clinicians in and around Guntur. International Journal of Recent Trends in Science And Technology 2012;5(2):100103.

12. Hanumantha Rao Potharaju, Kabra SG. Prescription audit of outpatient attendees of secondary level government hospitals in Maharashtra. Indian Journal Of Pharmacology 2011;43(2):150-6.

13. Yasmeen Maniyar, Prabhu Bhixavatimath, Vidyashree Akkone. A drug utilization study in the ophthalmology department of a medical college, Karnataka, India. Journal of clinical and diagnostic Research 2011;5(1):82-4.
14. Rataboli PV, Amit Garg. Confusing brand names: nightmare of medical profession. Journal of Post Graduate Medicine 2005;51(1):13-6.

15. Damodaran RT. Brand confusion causes allergic dermatitis. Journal of Post Graduate Medicine 2005;51(3):242.

16. Sudheer G, Ravi Prabhu G, Sridhar MS. A study of prescription writing practices of doctors in geriatric age group patients in a teaching hospital. Jour of Evolution of Medical and Dental Sciences 2015;4(3):322-8.

17. Mohan P, Sharma AK, Panwar SS. Identification and quantification of prescription errors. Medical journal Armed forces India 2014;70(2):149-53.

18. Daisy Wadhwa, Bharti Tomar, Harpreet Grewal, et al. Medication errors in dentistry-a cross-sectional study. Delhi psychiatric Journal 2014;17(1):107-12.

19. Manoj Kumar Saurabh, Niraj Kumar Biswas, Ashish Kumar Yadav, et al. Study of prescribing habits and assessment of rational use of drugs among doctors of primary health care facilities. Asian journal of pharmaceutical and clinical research 2011;4(4):102-5.

20. Vijayakumar TM, Sathyavati D, Subhashini T, et al. Assessment of prescribing trends and rationality of drug prescribing. International Journal of Pharmacology 2011;7(1):140-3. 